\title{
SINERGISMO PARA LA SOLUCIÓN DE PROBLEMAS AMBIENTALES: REMEDIACIÓN DE FUENTES HÍDRICAS MEDIANTE EL EMPLEO DE BASES DE SCHIFF
}

SYNERGISM FOR THE SOLUTION OF ENVIRONMENTAL PROBLEMS: REMEDIATION OF WATER SOURCES THROUGH THE USE OF SCHIFF BASES

Diego Quiroga*

https://orcid.org/0000-0001-5156-8200

Lili Dahiana Becerra**

https://orcid.org/0000-0002-3366-022X

Paola Borrego-Muñoz***

https://orcid.org/0000-0002-0024-9629

UNIVERSIDAD MILITAR NUEVA GRANADA

Recibido: 2 de septiembre de 2019

Aceptado: 24 de enero de 2020

DOl: https://doi.org/10.29097/2011-639X.300

\section{Resumen}

Las fuentes de agua potable, necesarias para garantizar la salud de los seres vivos y el medioambiente, son cada vez más escasas. Esta grave problemática exige el desarrollo de nuevas tecnologías eco-amigables que permitan el monitoreo de metales pesados, la prevención y, especialmente, la remediación de fuentes hídricas contaminadas. Dada la necesidad de orientar las investigaciones hacia el diseño de materiales capaces de retener o facilitar la eliminación de contaminantes con alta eficiencia y efectividad, en este artículo se presentan algunos de los últimos avances respecto al desarrollo de nuevos métodos de remediación de agua empleando bases de Schiff, una familia de moléculas de gran importancia en química. Los resultados de estas investigaciones demuestran su capacidad quelatante y como agente catalizador, en especial, su potencial aplicación para fines ambientales, siendo aún un conjunto de investigaciones incipientes, con mucho por explorar en este campo de la ciencia y la ingeniería.

Palabras clave: bases de Schiff, imina, enamina, remediación, fuentes de agua

Abstract

The sources of drinking water, necessary to guarantee the health of living beings and the environment, are increasingly scarce. This serious problem requires the development of new eco-friendly technologies which allow the heavy metals monitoring, prevention and, especially, contaminated water sources remediation. Understanding the necessity to lead the research activities towards the design of new materials which could

* Departamento de Química, Facultad de Ciencias Básicas y Aplicadas.

** Doctorado en Biociencias, Universidad de La Sabana.

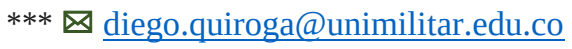


be promissory in retaining or facilitating pollutants removal with higher efficiency and effectiveness, this article presents some of the recent advances respect to the development of novel methods for water remediation employing Schiff bases, a family of molecules of great relevance in chemistry. The presented results allow to demonstrate both, the chelating and catalytic activities, and especially, the potential application for environmental purposes of Schiff bases; considering the latter, as an incipient research to explore still in Science and Engineering.

Keywords: schiff bases, imine, enamine, remediation, water resources.

\section{INTRODUCCIÓN}

El agua es uno de los recursos de mayor relevancia para la supervivencia de los ecosistemas vivos. El conjunto de investigaciones dirigido a la remediación de los sistemas hídricos corresponde a un área de trabajo importante dentro de las ciencias básicas y la ingeniería. El mayor nivel de consumo de agua y los altos niveles de contaminación asociados han generado una necesidad de gestionar su calidad y mantener niveles seguros de los contaminantes presentes, para que pueda ser empleada sin afectar la salud humana y del medio ambiente (Pescod, 1992; McIntosh \& Pontius, 2017). Son múltiples los iones metálicos que contaminan las diferentes fuentes hídricas, lo que conlleva a limitar e incluso a impedir su consumo, siendo desperdiciados millones de metros cúbicos de este importante recurso. Por ejemplo, la cuenca del río Tapajós, en la Amazonía brasileña, la cual ha sido objeto de estudio en varias investigaciones (Lino et al., 2019), presenta diversas actividades antropogénicas que aumentan las concentraciones de mercurio (Hg) en el ecosistema acuático, además de que han sido encontradas naturalmente altas concentraciones de este en dicha cuenca. Las distribuciones del mercurio total (THg) y metilado (MeHg) evaluadas en agua sin filtrar ( $n=47)$, partículas suspendidas (SPM, $n=30$ ), sedimento superficial (BS, $n=29$ ), plancton $(n=28)$ y peces $(n=129)$ de la cuenca del río Tapajós, evidenciaron que las partículas suspendidas fueron el principal portador de Hg en la columna de agua y los sedimentos; que el aumento de la erosión, provocado por las actividades antropogénicas, condujo a mayores concentraciones de $\mathrm{Hg}$ en el agua de las áreas más afectadas; y que el Hg se transporta principalmente en partículas, de tal forma que las perturbaciones antrópicas influyen en las concentraciones de Hg aguas abajo. La metilación de Hg en el plancton total fue más eficiente en lagos (13-66\%) que en el canal principal del río Tapajós (2-14\%), demostrándose que los factores bióticos y abióticos interactúan de manera compleja en el ecosistema acuático, lo que hace que las concentraciones de Hg varíen en la red alimentaria.

Además del monitoreo de metales pesados como Hg, los esfuerzos de prevención y remediación deben centrarse en el control de la erosión del suelo y los sedimentos. Este tipo de casos demuestra la importancia de orientar las investigaciones hacia el diseño de materiales capaces de retener iones metálicos pesados (como Hg), que propicien la remediación de fuentes hídricas con alta eficiencia y efectividad. Desde la conciencia de esta problemática, el desarrollo de nuevos métodos de remediación del agua ha dado un paso adelante para mejorar la calidad del agua potable y la del agua de grado industrial, para evitar la contaminación de los recursos hídricos naturales debido a la 
descarga de efluentes industriales. Varios métodos de remediación del agua (físicos, químicos, electroquímicos y biológicos) se discuten y evalúan en todo el mundo constantemente (Bhattacharya et al., 2018). Sin embargo, en pleno siglo XXI no se ha logrado establecer una estrategia estándar que permita remediar eficientemente esta situación. Lo anterior evidencia una problemática latente, real e impactante, la cual debe ser estudiada desde el punto de vista de la química ambiental para la consecución de una solución desde la ingeniería. Estas situaciones tan solo representan una pequeña fracción del problema en torno a la necesidad de establecer nuevas tecnologías que permitan una adecuada remediación de fuentes hídricas, indispensables para garantizar la sostenibilidad de la población mundial.

En ese sentido, diversas metodologías han mostrado el uso de ligandos tipo base de Schiff y/o complejos metálicos derivados para el diseño de nuevos materiales y metodologías que puedan ser empleadas en procesos de remediación. Adicionalmente, este grupo de moléculas ha ganado protagonismo al obtenerse una estimación de su potencial aplicación en otras problemáticas globales, enmarcadas en los objetivos de desarrollo sostenible de la ONU (2019). Su fácil síntesis y la exploración de su capacidad quelatante han permitido consolidar algunos avances en el diseño de materiales que puedan ser usados para remediación de fuentes hídricas de agua, campo de estudio de los últimos años con múltiples grupos de investigación a nivel mundial. Por lo anterior, en este artículo se presentan algunos de los más recientes avances en torno al uso de esta familia de moléculas, ampliamente conocida en química por su facilidad de síntesis y variada actividad biológica.

\section{GENERALIDADES DE LAS BASES DE SCHIFF}

Las bases de Schiff son compuestos que fueron reportados por primera vez por el químico alemán Hugo Schiff en 1864. Estos compuestos son convencionalmente obtenidos por condensación de aminas primarias y compuestos carbonílicos. La presencia de agua normalmente limita el rendimiento de la reacción. Sin embargo, a lo largo de los años han sido concebidas varias mejoras, todas orientadas a retirar el agua formada en el medio de reacción. Este propósito ha sido logrado empleando disolventes deshidratantes como ortosilicato de tetrametilo, ortoformiato de trimetilo, e incluso tamices moleculares y otros agentes que eliminan completamente el agua formada en el medio de reacción. Sin embargo, es conocido que la reacción depende del uso de compuestos carbonílicos altamente electrofílicos y aminas fuertemente nucleófilas, por lo que se han utilizado ácidos de Brönsted-Lowry o de Lewis como agentes catalíticos (Da Silva et al., 2011).

En los últimos años han sido empleadas varias alternativas para mejorar la estrategia de síntesis, las cuales están asociadas al uso de diversas fuentes de energía como la irradiación de microondas o el ultrasonido en ausencia de disolvente. Entre estas innovaciones, la irradiación de microondas se ha utilizado ampliamente debido a su simplicidad operativa, sus velocidades de reacción mejoradas y su gran selectividad (Pradhan et al., 2019). 
Quiroga, Becerra y Borrego-Muñoz

\section{Figura 1.}

Síntesis de iminas en dos pasos

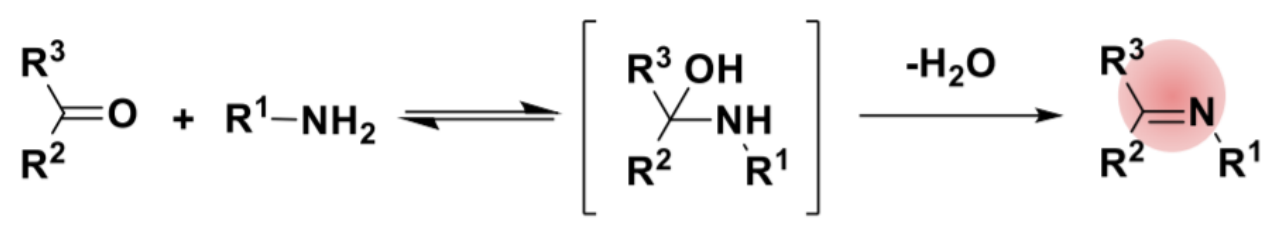

\section{$R^{1}, R^{2}, y / o R^{3}=$ alquil o aril}

Nota: Representación de la reacción general correspondiente a la síntesis de iminas en dos pasos, mostrando la formación de un intermediario de adición. Fuente: Chemdraw ${ }^{\circledR}$ Professional 19.0 suite Free Trial

Esta reacción ha sido ampliamente estudiada en la literatura, siendo aplicada en la mayoría de los casos para la obtención de iminas alifáticas. Sin embargo, dada la complejidad que representa la síntesis de sistemas anulares tipo imina, este método es limitado a anillos de cinco, seis y, en algunos casos, siete átomos. Así, reacciones pericíclicas como cicloadiciones, acoplamientos cruzados carbono-carbono empleando catálisis metalorgánica, y empleo de sistemas catalíticos heterogéneos, son metodologías poco conocidas en el contexto de la síntesis de bases de Schiff, pero con una proyección interesante dada su practicidad y aplicabilidad (Otero et al., 2011). Estructuralmente, las bases de Schiff están caracterizadas por la presencia de al menos un grupo imina $(\mathrm{C}=\mathrm{N})$, el cual usualmente presenta un comportamiento químico similar al mostrado por los compuestos carbonílicos, especialmente en lo que respecta a su reactividad. Además, en disolución suelen estar en equilibrio tautomérico, especialmente en bases de Schiff con hidrógenos activos en el átomo de carbono adyacente, de tal forma que coexisten dos formas tautoméricas: imina y enamina.

\section{Figura 2.}

Equilibrio tautomérico existente entre iminas y enaminas

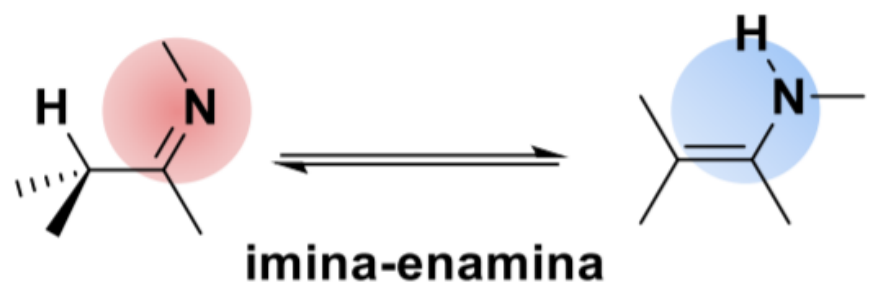

Nota: Representación del equilibrio tautomérico existente entre iminas y enaminas usando un esquema de reacción química. Fuente: Chemdraw ${ }^{\circledR}$ Professional 19.0 suite Free Trial 
Se ha demostrado que la forma imina es químicamente más estable que la forma enamina, sin bien la barrera energética para la interconversión a la forma enamina es de un orden de magnitud menor que la de la tautomerización ceto-enol (Basa et al., 2012). El desplazamiento del equilibrio tautomérico hacia la enamina en disolventes polares capaces de aceptar un protón y formar un enlace de hidrógeno intermolecular ha sido demostrado en varias investigaciones (Kostochka \& Lezina, 1994). Sistemas $N$ arilsustituidos tienden a sufrir un cambio análogo, con desplazamiento del equilibrio hacia la formación de la enamina, resultado de la conjugación del par de electrones del átomo de nitrógeno con el sistema aromático que extiende la conjugación del sistema $\pi$ extendido. De acuerdo con esto, dependiendo de las condiciones del medio en el cual se encuentren, puede llegarse a favorecer una de las estructuras tautoméricas descritas. La presencia de una amplia densidad electrónica tanto en la forma imina como enamina las hace comportarse como ligandos interesantes de metales pesados (Dalia et al., 2018).

La literatura científica cuenta con una gran cantidad de artículos publicados respecto a la síntesis de bases de Schiff y su uso como ligandos de metales pesados. Hand \& Jenks (1975) evidenciaron que las constantes de velocidad observadas para la ionización de nitroetano, y de bases de Schiff derivadas del mismo, aumentan linealmente con la concentración de acetato hasta 2,0 M cuando la fuerza iónica se mantiene con trifluoroacetato de potasio. Una pequeña curvatura hacia arriba cuando la fuerza iónica se mantiene con nitrato de potasio se atribuye a un efecto de sal específico. Se encuentra una curvatura hacia arriba más grande para la enolización de ciclohexanona tanto con trifluoroacetato como con nitrato, la cual se atribuye a la catálisis bifuncional ácidobase. No se encontraron pruebas de asociación de tampón para estas reacciones. Chow et al. (1977) describen la preparación de complejos de cobre (II) con una base de Schiff derivada de salicilaldehído y $L$-arginina. Los complejos de nitrato de cobre mostraron ser antiferromagnéticos, proponiendo una estructura tetramérica. Ambos complejos de níquel mostraron obedecer las leyes de Curie-Weiss con $\theta=20 \mathrm{~K}$ y $30 \mathrm{~K}$ para el nitrato y el cloruro. Sus bajos momentos magnéticos sugirieron que pueden ser mezclas de configuraciones octaédricas y planas cuadradas. Burk et al. (1994) describieron un procedimiento conveniente de aminación reductiva asimétrica quimioselectiva para la conversión de cetonas en hidrazinas y aminas quirales. El paso clave en el proceso de tres pasos fue la hidrogenación catalizada por DuPHOS-Rh enantioselectiva del doble enlace $\mathrm{C}=\mathrm{N}$ de las $\mathrm{N}$-acilhidrazonas. Los estudios detallados de optimización revelaron el efecto del solvente, la temperatura y el grupo $\mathrm{N}$-acilo sobre la enantioselectividad y la eficiencia catalítica de la reacción. Los productos de reducción, N-acilhidrazinas, se convirtieron en hidrazinas o aminas mediante hidrólisis o tratamiento con yoduro de samario. Teo et al. (1994) estudiaron reacciones de aminometilación de Mannich que involucran iones metálicos de zinc, níquel, cobre y cobalto, dando como resultado la formación de los respectivos complejos de metal y $N, N$-di- $N^{\prime}$-(metilacetamido)glicina como ligando.

Por otro lado, las bases de Schiff se han utilizado como sintones en la preparación de una serie de compuestos industriales y biológicamente activos como formazanos, 4tiazolidininas y benzoxazinas, entre otros, mediante el cierre del anillo, la cicloadición y las reacciones de reemplazo (Kajal et al., 2013). Los derivados de bases de Schiff obtenidos en procesos industriales han sido empleados en el diseño de nuevas bases con 
fragmentos heterocíclicos y aromáticos, en pro del desarrollo de nuevos sistemas moleculares y materiales con gran aplicabilidad en química e ingeniería.

También se ha demostrado que las bases de Schiff exhiben una amplia gama de actividades biológicas, que incluyen propiedades antifúngicas, antibacterianas, antipalúdicas, antiproliferativas, antiinflamatorias, antivirales y antipiréticas (Da Silva et al., 2011). Los grupos imina o azometina están presentes en varios compuestos naturales, derivados naturales y no naturales. Se ha demostrado que el grupo imina presente en tales compuestos es crítico para sus actividades biológicas. Sopena De Kracoff, De Sancovich \& Sancovich (1995) evidenciaron que la enzima ácido-5aminolevulínico-deshidratasa cataliza la autocondensación entre dos moléculas de ácido 5-aminolevulínico, a través de una base de Schiff en la que se propone involucrar un residuo de lisina en su sitio activo. Las bases de Schiff formadas entre el sustrato o el 5fosfato de piridoxal y la enzima se estabilizaron mediante reducción con $\mathrm{NaBH}_{4}$. El levulinato y el piruvato actuaron como inhibidores enzimáticos competitivos.

Casas et al. (2003) hicieron reaccionar 5'-fosfato de piridoxal con derivados de diorganoestaño (IV) en presencia y ausencia de aminoácidos. Los respectivos complejos se aislaron y caracterizaron, evidenciando que la base de Schiff fue formada presumiblemente por transaminación entre valina o glicina. La difracción de rayos $\mathrm{X}$ mostró que la estructura molecular consiste en moléculas en las que el ligando se une al metal a través de los átomos de oxígeno de los dos grupos hidroxilo fenólicos desprotonados y el átomo de nitrógeno imínico, y el metal exhibe una coordinación piramidal cuadrada distorsionada. Tanto las bases de Schiff como sus complejos mostraron una intensa actividad antibacteriana contra Pseudomonas aeruginosa, pero sólo los complejos exhiben actividad significativa contra las otras cuatro cepas bacterianas ensayadas, Staphylococcus aureus, Bacillus subtilis, Escherichia coli y una cepa de $P$. aeruginosa resistente a carbapenem.

\section{APLICACIÓN DE BASES DE SCHIFF PARA EL DISEÑO DE MATERIALES CON POTENCIAL USO EN REMEDIACIÓN DE FUENTES HÍDRICAS}

Se ha reportado el uso de bases de Schiff en aplicaciones de química analítica, bioorgánica, inorgánica y biología. El uso de estos compuestos como ligandos de complejos metalorgánicos ha sido demostrado ampliamente. Estos complejos han sido empleados como reactivos cromogénicos para uso en técnicas espectrofotométricas, y como intercambiadores de iones en métodos cromatográficos y/o ionóforos en la preparación de electrodos selectivos de iones. Se han desarrollado métodos espectrofotométricos para la determinación del ion $\mathrm{Ni(II)}$ empleando $N$-[2-tienilmetiliden]aminopropano (imina derivada del 2-tiofencarbaldehído con aminopropano) como agente complejante, e incluso aplicaciones como electroforesis capilar (EC), cromatografía líquida y de gases han mostrado la aplicabilidad de estos compuestos (Pleniceanu, Spinu \& Isvoranu, 2007; Memon et al., 2014). Por lo anterior, esta amplia familia de moléculas resulta de interés particular en química ambiental, y su aplicabilidad abre nuevos paradigmas dado su potencial uso. Con todo, el planteamiento y ejecución de los métodos de síntesis de este tipo de sistemas está condicionado en gran medida a diferentes consideraciones estructurales, que pueden ser aprovechadas 
para el diseño de nuevos materiales (Berhanu et al., 2019). Recientemente se ha desarrollado un adsorbente compuesto de un biopolímero, mediante la introducción de montmorillonita modificada con sulfhidrilo en una matriz de carboximetilquitosano funcionalizada con polietilenimina hiperramificada (HPFC / MT-S), que demostró ser una alternativa superior para la eliminación de iones Hg (II) de soluciones acuosas (Zeng et al., 2019). Sin embargo, es indispensable profundizar en las diversas investigaciones que propenden por el diseño de materiales capaces de retener iones metálicos contaminantes.

El desarrollo de carbocatalizadores altamente activos y multifuncionales modificados con heteroátomos o especies metálicas es crucial para aplicaciones prácticas de remediación ambiental. El carbono poroso dopado con nitrógeno embebido con nanodots de óxido de cobalto altamente dispersos (CoO-N-C) fue obtenido a partir de un polímero base de Schiff derivado de biomasa. El catalizador de CoO-N-C exhibió una excelente eficiencia de adsorción de 4-CP, así como un excelente rendimiento catalítico en la activación de peroximonosulfato (PMS) para la degradación de 4-CP. La eliminación total de carbono orgánico (TOC) fue cercana al 99,7\% e implicó una combinación de procesos de adsorción y degradación, siendo un efecto sinérgico entre los nanodots de $\mathrm{CoO}$ integrados y el nitrógeno dopado para el aumento de la densidad de electrones en la superficie de carbono del catalizador, acelerando así el proceso de transferencia de electrones para la activación de PMS y mejorando el rendimiento catalítico (Xie et al., 2020).

Por otro lado, a partir del acoplamiento de la hidrazona derivada de rodamina fue obtenida una sonda altamente selectiva y sensible a iones $\mathrm{Hg}^{2+}$, a través de la reacción de hidrólisis específica de los éteres de vinilo con un apagado de fluorescencia significativo en solución tampón de $\mathrm{CH}_{3} \mathrm{CN}-\mathrm{PBS}$ (3:7, v/v). La sonda también mostró detección fluorescente para $\mathrm{Cu}^{2+}$ en muestras contaminadas (Dong et al., 2020).

Las bases de Schiff también han sido empleadas en el diseño de materiales ecológicos para detectar metales pesados y disminuir las contaminaciones en aguas naturales. La celulosa fue modificada con un Bodipy para realizar mediciones espectroscópicas útiles que permitieran detectar y eliminar eficazmente el ion Fe (III) del medio acuoso. La celulosa a base de Bodipy (Cell-BODIPY) exhibió un rendimiento excepcional para detectar iones Fe (III) bajo luz de onda larga y luz de día. Para una mejor visualización en aplicaciones prácticas, también se realizó el mismo proceso de modificación para papel de filtro, obteniendo muy buenos resultados (Oguz, Kursunlu \& Yilmaz, 2020). Los materiales mesoporosos SBA-15, funcionalizados con la base de Schiff derivada de 2-acetiltiofeno denotada como A-SBA-15, se diseñaron para la eliminación de iones Cr (III) en solución acuosa, mostrando una capacidad máxima de adsorción de A-SBA-15 (114,2 mg/g) para iones Cr (III) mucho mayor que la del SBA15 puro (15,6 mg/g). La adsorción de Cr (III) en el material A-SBA-15 es espontánea y endotérmica, y ocurre a través de la formación de enlaces de coordinación. Este material (A-SBA-15) puede reutilizarse en cinco ciclos sucesivos sin disminuir significativamente su capacidad de adsorción y puede ser preparado en diferentes tiempos de síntesis manteniendo una capacidad de adsorción similar. Además, ha demostrado una buena separación selectiva de iones $\mathrm{Cr}$ (III) en presencia de iones $\mathrm{Cu}$ (II), Zn (II) y Ni (II). La buena reproducibilidad, reutilización y selectividad han 
sugerido que el material A-SBA-15 puede usarse como un adsorbente prometedor para la eliminación eficiente de metales pesados de una solución acuosa (Wu et al., 2020; Chen et al., 2019).

La adsorción es, hasta la fecha, la tecnología más efectiva y utilizada a nivel mundial para eliminar varios contaminantes en las aguas residuales (Renu, Agarwal \& Singh, 2017). Muchos adsorbentes se han sintetizado, probado y utilizado para la eliminación y separación de contaminantes como radionúclidos, metales pesados, colorantes y compuestos farmacéuticos, tanto a escala de laboratorio como industrial. Dos métodos que han mostrado resultados prometedores y han ganado un interés significativo son el uso de nanomateriales magnéticos y de biosorbentes, debido a sus propiedades efectivas, seguras, ecológicas, de bajo costo y de bajo consumo de energía (Maksoud et al., 2020). Nuevos sorbentes bioconjugados, péptidos y oligonucleótidos en nanomateriales de aluminato de cobalto, diseñados para eliminar formas catiónicas de plomo, fueron empleados para la remediación de fuentes hídricas. Después de la optimización de los parámetros experimentales, se obtuvo una separación exitosa a pH $7,5$, con una recuperación cuantitativa alta ( $>95 \%)$ y una alta precisión $(<10 \%$ de desviación estándar relativa). Usando los sorbentes bioconjugados propuestos, el plomo en las muestras de agua de mar y agua de grifo podría ser eliminado práctica y fácilmente con un nivel de confianza del 95\% (Morcali \& Baysal, 2019). También se han obtenido nanopartículas de $\mathrm{Fe}_{2} \mathrm{O}_{3}$ (hematita) con diferentes tamaños de cristal (40$59 \mathrm{~nm}$ ) a partir de latas de insecticidas (Abdelrahman et al., 2019). Los nanomateriales magnéticos actúan como adsorbentes eficientes debido a su carga superficial, a su actividad redox y a su facilidad para eliminar contaminantes del agua cuando se aplica un campo magnético, ayudando de esta manera a aliviar problemas ecológicos y ambientales (Gul et al., 2019).

En estas dos rutas prometedoras también han sido usadas bases de Schiff como ligandos de metales pesados. Se ha demostrado que la presencia de estas facilita la adsorción, mejorando su eficiencia, reutilización y practicidad para la eliminación de contaminantes de corrientes de aguas residuales (Maksoud et al., 2020). Materiales poliméricos porosos (PPM) con ligandos de óxido de fosfina/calcogenuro fueron empleados para la captura eficiente de yodo de la fase de vapor, usando óxido/sulfuro/seleniuro de trifenilfosfina e introduciendo ligandos tipo base de Schiff en el esqueleto de silsesquioxano oligomérico poliédrico (Zhang et al., 2020). Otros materiales mesoporosos adsorbentes son altamente eficientes para aplicaciones ambientales, dado que los contaminantes se eliminan mediante el proceso de adsorción tradicional. La síntesis de materiales mesoporosos basados en MCM-41 y su uso estratégico como material adsorbente para la eliminación de diferentes contaminantes ha sido objeto de estudio en los últimos años (Costa et al., 2020). Recientemente se han evaluado los principales factores que afectan su síntesis, y especialmente, los métodos de funcionalización orientados hacia la preparación de materiales mesoporosos basados en MCM-41. Este tipo de tecnología de adsorción ha atraído mucha atención, debido a que es una técnica que requiere el uso de pocos pasos y equipos, es de fácil operación y bajo costo, no genera residuos ni subproductos, es ecológica y de alta eficiencia energética, lo que permite su amplia aplicación en los procesos de remediación (Shields et al., 2020). Polioxometalatos de tipo Keggin H3PW12O40 (PW12), H3PMo12O40 
(PMo12) y H4SiW12O40 (SiW12), soportados en polianilina (PANI) recubierta con nanopartículas de CuS (lo cual se realiza mediante un método de modificación gradual sencillo), fueron elaborados y evaluados como dispositivos de adsorción de azul de metileno (MB) y naranja de metilo (MO) (Siadatnasab, Karami \& Khataee, 2019). Estos materiales exhibieron una alta selectividad y una alta tasa de adsorción para MB catiónico en lugar de MO aniónico. Por otro lado, el diseño y la síntesis de polímeros porosos conjugados basados en BOPHY (IEP-7 e IEP-8) ha mostrado actividad fotocatalítica heterogénea bajo luz visible. Estos polímeros porosos también mostraron actividad como fotocatalizadores eficientes en la oxidación selectiva de varios sulfuros hacia sulfóxidos, menos nocivos para el medioambiente (López-Calixto et al., 2019). Materiales similares han sido empleados para la síntesis de nanopartículas de plata combinadas con óxido de itrio $\left(\mathrm{Ag}-\mathrm{Y}_{2} \mathrm{O}_{3}\right)$, utilizando úrea como material combustible. Estos nanocompuestos mostraron ser térmicamente estables, asimétricos y bien distribuidos sin ninguna agregación, además de que su aplicabilidad para la eliminación múltiple de metales pesados de soluciones acuosas se probó realizando experimentos para $\mathrm{Cu}$ (II) y $\mathrm{Cr}$ (VI), evidenciando una reducción en el porcentaje de eliminación de Cu (II) (98,5 a 93\%) y Cr (VI) (98,3 a 95,1\%) (Pradhan et al., 2019). La síntesis hidrotérmica de un producto de valor agregado del ácido metacrílico (MAA), realizada a través de la decoxilación catalizada por Co (II) de ácido itacónico (IA), junto con la formación de dos Co (II)-MOFs basados en algunas bases de Schiff, ha permitido evidenciar que los MOFs son estables al agua, fluorescentes y exhiben una estructura de detección de cationes regulados como $\mathrm{Fe}^{3+} \mathrm{y} \mathrm{Hg}^{2+}$ con una selectividad muy alta, sensibilidad entre 10-100 ppb y buena reutilización (Thakur, Pandey \& Pandey, 2019). Sin embargo, la recuperación de iones metálicos de soluciones acuosas para la preservación del medio ambiente o el ahorro de recursos requiere el desarrollo de nuevos sorbentes con propiedades de separación mejoradas.

Estos antecedentes han mostrado la importancia del diseño y la funcionalización de nuevos materiales, como micropartículas de quitosano con injerto de bases de Schiff, amidoxima (AO-AHC) o hidrazinilamina (HZ-AHC) (Hamza et al., 2019). La fabricación de un adsorbente novedoso, con una gran área superficial y una alta capacidad de adsorción, es una demanda urgente para el tratamiento del agua. Nanocompuestos que incluyen bases de Schiff y nanopartículas magnéticas de $\mathrm{Fe}_{3} \mathrm{O}_{4}$ han sido sintetizados y su actividad ha sido estudiada. Un polímero orgánico poroso magnético (MPOP) fabricado fue usado como un adsorbente eficiente para la eliminación de Cd (II) y Hg (II) de soluciones acuosas. Los resultados termodinámicos indicaron que la adsorción de ambos iones metálicos fue espontánea y endotérmica. Los resultados de la regeneración sugirieron que el nuevo MPOP era un adsorbente reutilizable prometedor para la adsorción de iones de metales pesados de una solución acuosa (Alhokbany et al., 2019). Otras nanopartículas obtenidas a partir de úrea, glicina, $L$-alanina y $L$-valina fueron evaluadas contra cristal violeta (CV) y azul de metileno (MB), siendo eliminados estos contaminantes eficientemente de la solución acuosa mediante degradación fotocatalítica bajo irradiación UV en presencia de $\mathrm{Fe}_{2} \mathrm{O}_{3}$ y $\mathrm{H}_{2} \mathrm{O}_{2}$ (Abdelrahman et al., 2019). El uso de materiales para procesos redox orientados en la remediación de muestras hídricas también ha sido explorado (Deng et al., 2019). La síntesis de un ligando base de Schiff diácido mediante la reacción de 2-hidroxibenzaldehído con ácido 5-aminoisoftálico, así como su posterior uso en la síntesis de una nanoestructura 
mediante la reacción de 4,4'-diaminodifenil éter y el diácido sintetizado en presencia de bromuro de tetrabutilamonio fundido (ТВAB)/fosfito de trifenilo (TPP), son otros ejemplos del uso de estas moléculas. El complejo de poliamida mostró un buen comportamiento de adsorción para la eliminación de iones Cd (II) y Hg (II) de las soluciones acuáticas, además der ser fácil de recuperar y reutilizar (Aghaei, Kianfar \& Dinari, 2019; Li et al., 2019; Abu Elella et al., 2019).

En general, los resultados demuestran que las capacidades máximas de adsorción dependen del metal y del tipo de funcionalización. El pequeño tamaño de las partículas magnéticas limita la contribución de los mecanismos de difusión en el control general de la cinética de adsorción o absorción y en el tiempo requerido para lograr el equilibrio, demostrando que la creación de nuevas tecnologías para la remediación de fuentes hídricas es un área con mucho por explorar aún, y que el desarrollo de nuevas tecnologías en dicho campo conducirá a un mejoramiento en la calidad de vida de los seres vivos.

\section{APLICACIONES DIRIGIDAS A LA SOLUCIÓN DE OTRAS PROBLEMÁTICAS AMBIENTALES}

La captura y transformación simultánea de dióxido de carbono de los efluentes industriales en productos químicos de valor agregado se considera una de las tecnologías más prometedoras para la valorización del $\mathrm{CO}_{2}$ en productos renovables. La carboxilación de intermedios industriales como epóxidos, alcoholes y polioles en carbonatos orgánicos ha sido el foco de grandes esfuerzos de investigación, ya que proporciona nuevas rutas sintéticas para productos esenciales para la sociedad humana. Sin embargo, la conversión selectiva de $\mathrm{CO}_{2}$ a carbonatos en condiciones leves sigue siendo un gran desafío. El diseño racional de materiales catalíticos homogéneos y heterogéneos, con una discusión exhaustiva y crítica sobre la síntesis del catalizador y las estructuras y mecanismos de reacción plausibles, ha sido un tópico ampliamente estudiado en los últimos cinco años (Jin et al., 2019).

En química analítica, algunos sensores colorimétricos basados en fibra de pulpa de biomasa (PF), injertando $L$-lisina ( $L$-lys) sobre su superficie, fueron obtenidos mediante una reacción de tipo Schiff. El injerto $L$-lys que contenía dos grupos específicos de reconocimiento sinérgico $\left(-\mathrm{COOH}\right.$ y $-\mathrm{NH}_{2}$ ), mostró un excelente reconocimiento selectivo de $\mathrm{Ag}^{+}$y un cambio de color visual evidente, de amarillo claro a marrón, en soluciones acuosas que contienen otros iones $\mathrm{Cu}^{2+}, \mathrm{Pb}^{2+}, \mathrm{Mn}^{2+}, \mathrm{Mg}^{2+}, \mathrm{Cd}^{2+}, \mathrm{Ca}^{2+}, \mathrm{H}_{2} \mathrm{O}$, $\mathrm{Al}^{3+}, \mathrm{Hg}^{2+}, \mathrm{Cr}^{6+}, \mathrm{K}^{+}, \mathrm{Na}^{+}$y $\mathrm{Zn}^{2+}$, exhibiendo perspectivas prometedoras para la identificación in situ de $\mathrm{Ag}^{+}(\mathrm{ppb})$, debido a su alta selectividad y sensibilidad (Zhu et al., 2019).

El nitruro de carbono es un prometedor semiconductor libre de metales orgánicos poliméricos conjugados con $\pi$. Dado su potencial uso en síntesis química, su estabilidad química y su estructura electrónica, ha despertado un enorme interés en la fotocatálisis. Varios esfuerzos han sido dirigidos para superar algunas limitaciones estructurales de los materiales poliméricos mediante el diseño de la estructura electrónica de nitruro de carbono a nivel molecular. Una de las formas conlleva a la realización de 
modificaciones racionales en la estructura electrónica de g- $\mathrm{C}_{3} \mathrm{~N}_{4}$ conjugado mediante la integración de un conjunto diversificado de fragmentos orgánicos a través de enlaces covalentes con algunas bases de Schiff, lo cual ha demostrado ser promisorio. Dicha funcionalización proporciona una fuerte interacción covalente entre fragmentos funcionalizados, mejorando sinérgicamente la estabilidad, así como las propiedades electrónicas y ópticas (Chauhan et al., 2019). Otro de los grandes retos ambientales surgió con el rápido desarrollo y aplicación de la energía y la tecnología nuclear, lo cual produjo una gran cantidad de residuos radiactivos. El óxido de grafeno y sus compuestos han atraído mucha atención en el campo del tratamiento de residuos radiactivos, debido a su alta superficie específica, abundantes grupos funcionales, fuerte capacidad de adsorción y buena estabilidad química (Ali et al., 2019). La adsorción de radionucleidos por el óxido de grafeno y sus compuestos, el estudio de su capacidad de adsorción, el modelo isotérmico de adsorción, la termodinámica de adsorción, los factores de influencia y el mecanismo de adsorción del óxido de grafeno y sus compuestos para radionucleidos han sido ampliamente estudiados (Liu et al., 2019), mostrando resultados promisorios, pero además muchos retos aún por vencer de parte de la comunidad científica.

\section{CONCLUSIONES}

El desarrollo de nuevas tecnologías ha traído consigo una gran cantidad de problemáticas ambientales. La comunidad científica ha centrado esfuerzos para resolverlas, dirigiendo sus investigaciones a la remediación ambiental. Las investigaciones dirigidas al uso de materiales adsorbentes modificados han crecido en los últimos años, dentro de las cuales las bases de Schiff han jugado un papel relevante por su estructura electrónica. A partir de los antecedentes recientes, se concluye que deben centrarse los esfuerzos para consolidar el desarrollo de tecnologías que aporten en la exigente tarea de lograr una solución de estas problemáticas ambientales, consolidando el ejercicio de la ciencia en un quehacer eco-sostenible e innovador, que garantice la preservación del medio ambiente.

\section{Referencias}

Abdelrahman, E. A., Hegazey, R. M., Kotp, Y. H., \& Alharbi, A. (2019). Facile synthesis of $\mathrm{Fe}_{2} \mathrm{O}_{3}$ nanoparticles from Egyptian insecticide cans for efficient photocatalytic degradation of methylene blue and crystal violet dyes. Spectrochimica Acta - Part A: Molecular and Biomolecular Spectroscopy, 222. 117195. doi: 10.1016/j.saa.2019.117195

Abu Elella, M. H., ElHafeez, E. A., Goda, E. S., Lee, S., \& Yoon, K. R. (2019). Smart bactericidal filter containing biodegradable polymers for crystal violet dye adsorption. Cellulose, 26 (17), 9179-9206. doi: 10.1007/s10570-019-02698-1

Aghaei, M., Kianfar, A. H., \& Dinari, M. (2019). Green synthesis of nanostructure Schiff base complex based on aromatic polyamide and manganese(III) for elimination of $\mathrm{Hg}(\mathrm{II})$ and $\mathrm{Cd}(\mathrm{II})$ from solutions. Journal of the Iranian Chemical Society, 16 (11), 2489-2500. doi: 10.1007/s13738-019-01719-x 
Alhokbany, N., Ahamad, T., Naushad, M., \& Alshehri, S. M. (2019). Feasibility of toxic metal removal from aqueous medium using Schiff-base based highly porous nanocomposite: Adsorption characteristics and post characterization. Journal of Molecular Liquids, 294. 111598. doi: 10.1016/j.molliq.2019.111598

Ali, I., Basheer, A. A., Mbianda, X. Y., Bukarov, A., Galunin, E., Burakova, I., Mkrtchyan, E., Tkachev, A., \& Grachev, V. (2019). Graphene based adsorbents for remediation of noxious pollutants from wastewater. Environment International, 127, 160-180. doi: 10.1016/j.envint.2019.03.029

Basa, P. N., Bhowmick, A., Horn, L. M., \& Sykes, A. G. (2012). Zinc(II) mediated imine-enamine tautomerization. Organic Letters, 14 (11), 2698-2701. doi: 10.1021/ol300874c

Berhanu, A. L., Gaurav, Mohiuddin, I., Malik, A. K., Aulakh, J. S., Kumar. V., \& Kim, K.-H. (2019). A review of the applications of Schiff bases as optical chemical sensors. TrAC Trends in Analytical Chemistry, 116, 74-91. doi: 10.1016/j.trac.2019.04.025

Bhattacharya, S., Gupta, A. B., Gupta, A., \& Pandey, A. (2018). Introduction to water remediation: importance and methods. doi: 10.1007/978-981-10-7551-3_1

Burk, M. J., Martinez, J. P., Feaster, J. E., \& Cosford, N. (1994). Catalytic asymmetric reductive amination of ketones via highly enantioselective hydrogenation of the CN double bond. Tetrahedron, 50 (15), 4399-4428. doi: 10.1016/S00404020(01)89375-3

Casas, J. S., Castiñeiras, A., Condori, F., Couce, M. D., Russo, U., Sánchez, A., Seoane, R., Sordo, J., \& Varela, J. M. (2003). Diorganotin(IV)-promoted deamination of amino acids by pyridoxal: SnR22+ complexes of pyridoxal 5'-phosphate and of the Schiff base pyridoxal-pyridoxamine (PLPM), and antibacterial activities of PLPM and [SnR2(PLPM-2H)] (R = Me, Et, Bu, Ph). Polyhedron, 22 (1), 53-65. doi: 10.1016/S0277-5387(02)01331-1

Chauhan, D. K., Jain, S., Battula, V. R., \& Kailasam, K. (2019). Organic motif's functionalization via covalent linkage in carbon nitride: An exemplification in photocatalysis. Carbon, 152, 40-58. doi: 10.1016/j.carbon.2019.05.079

Chen, J., Zhou, X., Sun, P., Zhang, Y., \& Huang, C.-H. (2019). Complexation Enhances $\mathrm{Cu}(\mathrm{II})$-Activated Peroxydisulfate: A Novel Activation Mechanism and Cu(III) Contribution. Environmental Science and Technology, 53 (20), 11774-11782. doi: 10.1021/acs.est.9b03873

Chow, S.-T., Johns, D. M., McAuliffe, C. A., \& Machin, D. J. (1977). Metal complexes of aminoacids and their derivatives. Part XIV. Magnetic and spectroscopic studies of complexes of copper(II) and nickel(II) with the Schiff base Nsalicylidenearginine. Inorganica Chimica Acta, 22, 1-5. doi: 10.1016/S00201693(00)90889-2

Costa, J. A. S., De Jesus, R. A., Santos, D. O., Mano, J. F., Romão, L. P. C., \& Paranhos, C. M. (2020). Recent progresses in the adsorption of organic, inorganic, and gas compounds by MCM-41-based mesoporous materials. Microporous and Mesoporous Materials, 291. 109698. doi: 10.1016/j.micromeso.2019.109698

Da Silva, C. M., Da Silva, D. L., Modolo, L. V., Alves, R. B., De Resende, M. A., Martins, C. V. B., \& De Fátima, A. (2011). Schiff bases: A short review of 
their antimicrobial activities. Journal of Advanced Research, 2 (1), 1-8. doi: 10.1016/j.jare.2010.05.004

Dalia, S. A., Afsan, F., Hossain, Md. S., Khan, Md. N., Zakaria, C. M., Kudrat-E-Zahan, Md., \& Ali, Md. M. (2018). A short review on chemistry of schiff base metal complexes and their catalytic application. International Journal of Chemical Studies, 6 (3): 2859-2866.

Deng, F., Li, S., Zhou, M., Zhu, Y., Qiu, S., Li, K., Ma, F., \& Jiang, J. (2019). A biochar modified nickel-foam cathode with iron-foam catalyst in electro-Fenton for sulfamerazine degradation. Applied Catalysis B: Environmental, 256. 117796. doi: 10.1016/j.apcatb.2019.117796

Dong, M., Tang, J., Lv, Y., Liu, Y., Wang, J., Wang, T., Bian, J., \& Li, C. (2020). A dual-function fluorescent probe for $\mathrm{Hg}$ (II) and $\mathrm{Cu}$ (II) ions with two mutually independent sensing pathways and its logic gate behavior. Spectrochimica Acta - Part A: Molecular and Biomolecular Spectroscopy, 226. 117645. doi: 10.1016/j.saa.2019.117645

Gul. S., Khan, S. B., Rehman, I. U., Khan, M. A., \& Khan, M. I. (2019). A Comprehensive Review of Magnetic Nanomaterials Modern Day Theranostics. Frontier Materials, 6, 179. doi: 10.3389/fmats.2019.00179

Hamza, M. F., Gamal, A., Hussein, G., Nagar, M. S., Abdel-Rahman, A. A. H., Wei, Y., \& Guibal, E. (2019). Uranium(VI) and zirconium(IV) sorption on magnetic chitosan derivatives - effect of different functional groups on separation properties. Journal of Chemical Technology and Biotechnology, 94 (12), 3866-3882. doi: 10.1002/jctb.6185

Hand, E. S., \& Jencks, W. P. (1975). Nonlinearity in Buffer-Rate Relationships. The Significance of Carboxylate-Acid Complexes. Journal of the American Chemical Society, 97 (21), 6221-6230. doi: 10.1021/ja00854a047

Jin, X., DIng, J., Xia, Q., Zhang, G., Yang, C., Shen, J., Subramaniam, B., \& Chaudhari, R. V. (2019). Catalytic conversion of $\mathrm{CO}_{2}$ and shale gas-derived substrates into saturated carbonates and derivatives: Catalyst design, performances and reaction mechanism (2019). Journal of $\mathrm{CO}_{2}$ Utilization, 34, 115-148. doi: 10.1016/j.jcou.2019.05.024

Kajal, A., Bala, S., Kamboj, S., Sharma, N., \& Saini, V. (2013). Schiff Bases: A Versatile Pharmacophore. Journal of Catalysts, Article ID 893512, 1-14. doi: 10.1155/2013/893512

Kostochka, L., \& Lezina, V. (1994). Imine-Enamine Tautomerism of Tropanone Schiff's Bases. Chemistry of Heterocyclic Compounds, 30 (3), 335-339. doi: 10.1007/BF01165701

Li, Y., Guo, C., Shi, R., Zhang, H., Gong, L., \& Dai, L. (2019). Chitosan/ nanofibrillated cellulose aerogel with highly oriented microchannel structure for rapid removal of $\mathrm{Pb}$ (II) ions from aqueous solution. Carbohydrate Polymers, 223. 115048. doi: 10.1016/j.carbpol.2019.115048

Lino, A. S., Kasper, D., Guida, Y. S., Thomaz, J. R., \& Malm, O. (2019). Total and methyl mercury distribution in water, sediment, plankton and fish along the Tapajós River basin in the Brazilian Amazon. Chemosphere, 235, 690-700. doi: 10.1016/j.chemosphere.2019.06.212 
Liu, H.-J., Wu, R.-J., Xie, S.-B., \& Liu, Y.-J. (2019). Graphene oxide and its composites for adsorption of radionuclides in water. Journal of Materials Engineering, 47 (10), 22-32. doi: 10.11868/j.issn.1001-4381.2018.001369

López-Calixto, C. G., Cabrera, S., Pérez-Ruiz, R., Barawi, M., Alemán, J., De la Peña O'Shea, V. A., \& Liras, M. (2019). Conjugated porous polymer based on BOPHY dyes as photocatalyst under visible light. Applied Catalysis B: Environmental, 258. 117933. doi: 10.1016/j.apcatb.2019.117933

Maksoud, M. I. A., Elgarahy, A. M., Farrell, C., Al-Muhtaseb, A. H., Rooney, D. W., \& Osman, A. I. (2020). Insight on water remediation application using magnetic nanomaterials and biosorbents. Coordination Chemistry Reviews, 403. 213096. doi: 10.1016/j.ccr.2019.213096

McIntosh, A., \& Pontius, J. (2017). Science and the Global Environment. Case Studies for Integrating Science and the Global Environment. Elsevier

Memon, S., Memon, N., Mallah, A., Soomro, R., \& Khuhawar, M. (2014). Schiff Bases as Chelating Reagents for Metal Ions Analysis. Current Analytical Chemistry, 10 (3), 393-417. doi: 10.2174/157341101003140521113731

Morcali, M. H., \& Baysal, A. (2019). The miniaturised process for lead removal from water samples using novel bioconjugated sorbents. International Journal of Environmental Analytical Chemistry, 99 (14), 1397-1414. doi: 10.1080/03067319.2019.1622695

Oguz, M., Kursunlu, A. N., \& Yilmaz, M. (2020). Low-cost and environmentally sensitive fluorescent cellulose paper for naked-eye detection of Fe (III) in aqueous media. Dyes and Pigments, 173. 107974. doi: 10.1016/j.dyepig.2019.107974

Organización de las Naciones Unidas (ONU). Objetivos de Desarrollo Sostenible. https://www.un.org/sustainabledevelopment/sustainable-development-goals/

Otero, A., Chapela, M.-J., Atanassova, M., Vieties, J. M., \& Cabado, A. G. (2011). Cyclic Imines: Chemistry and Mechanism of Action: A Review. Chemical Research in Toxicology, 24 (11), 1817-1829. doi: 10.1021/tx200182m.

Pescod, M. B. (1992). Wastewater treatment and use in agriculture. Food and Agriculture Organization of the United Nations.

Pleniceanu, M., Spinu, C., \& Isvoranu, M. (2007). Spectrophotometric Study of the Binary System Ni(II)-n-[2- Thyenilmethyliden]-Aminopropane and the Determination of Ni(II). Analytical Applications. Revista de Chimie, 57 (7), 646-649.

Pradhan, S. K., Pareek, V., Panwar, J., \& Gupta, S. (2019). Synthesis and characterization of ecofriendly silver nanoparticles combined with yttrium oxide $\left(\mathrm{Ag}-\mathrm{Y}_{2} \mathrm{O}_{3}\right)$ nanocomposite with assorted adsorption capacity for $\mathrm{Cu}(\mathrm{II})$ and $\mathrm{Cr}(\mathrm{VI})$ removal: A mechanism perspective. Journal of Water Process Engineering, 32. 100917. doi: 10.1016/j.jwpe.2019.100917

Renu, Agarwal M., \& Singh, K. (2017). Heavy metal removal from wastewater using various adsorbents: a review. Journal of Water Reuse and Desalination, 7 (4), 387-419. doi: 10.2166/wrd.2016.104

Shields, D. J., Chakraborty, M., Abdelaziz, N., Duley, A., \& Gudmundsdottir, A. D. (2020). Review of laser flash photolysis of organic molecules (2015-2018). Photochemistry, 47, 70-121. doi: 10.1039/9781788016520-00070 
Siadatnasab, F., Karami, K., \& Khataee, A. (2019). Keggin-type polyoxometalates supported on PANI-coated CuS: Synthesis, characterization and application as the efficient adsorbents for selective dye removal. Journal of Industrial and Engineering Chemistry, 80, 205-216. doi: 10.1016/j.jiec.2019.07.050

Sopena De Kracoff, Y. E., De Sancovich, A. M. F., \& Sancovich, H. A. (1995). Evidence of an essential lysine in pig liver 5-aminolevulinic acid dehydratase. International Journal of Biochemistry and Cell Biology, 27 (12), 1331-1339. doi: 10.1016/1357-2725(95)00097-9

Teo, S.-B., Ng, C.-H., Teoh, S.-G., \& Wei, C. (1994). Metal (II) complexes of (N,N-di$\mathrm{N}$-methylacetamido)glycine derived from mannich aminomethylation of bis(glycinato)metal(II), formaldehyde and acetamide. Polyhedron, 13 (17), 2537-2542. doi: 10.1016/S0277-5387(00)83096-X

Thakur, N., Pandey, M. D., \& Pandey, R. (2019). Co(II)-catalyzed decarboxylation of itaconic acid engendering methacrylic acid and Co(II)-MOFs for structure regulated fluorescent detection of cations. Journal of Solid State Chemistry, 280. 120987. doi: 10.1016/j.jssc.2019.120987

Wu, H., Xiao, Y., Guo, Y., Miao, S., Chen, Q., \& Chen, Z. (2020). Functionalization of SBA-15 mesoporous materials with 2-acetylthiophene for adsorption of Cr(III) ions. Microporous and Mesoporous Materials, 292. 109754. doi: 10.1016/j.micromeso.2019.109754

Xie, M., Tang, J., Fang, G., Zhang, M., Kong, L., Zhu, F., Ma, L., Zhou, D., \& Zhan, J. (2020). Biomass Schiff base polymer-derived N-doped porous carbon embedded with $\mathrm{CoO}$ nanodots for adsorption and catalytic degradation of chlorophenol by peroxymonosulfate. Journal of Hazardous Materials, 384. 121345. doi: 10.1016/j.jhazmat.2019.121345

Zeng, H., Wang, L., Zhang, D., Wang, F., Sharma, V. K., \& Wang, C. (2019). Amidofunctionalized carboxymethyl chitosan/montmorillonite composite for highly efficient and cost-effective mercury removal from aqueous solution. Journal of Colloid and Interface Science, 554, 479-487. doi: 10.1016/j.jcis.2019.07.029

Zhang, W., Mu, Y., He, X., Chen, P., Zhao, S., Huang, C., Wang, Y., \& Chen, J. (2020). Robust porous polymers bearing phosphine oxide/chalcogenide ligands for volatile iodine capture. Chemical Engineering Journal, 379. 122365. doi: 10.1016/j.cej.2019.122365

Zhu, H., Guo, W., Wang, J., He, H., Hou, X., Zhou, S., \& Wang, S. (2019). Tuneable design of a pulp fibre-based colorimetric sensor and its visual recognition mechanism for ppb levels of $\mathrm{Ag}^{+}$. Cellulose, 26 (17), 9149-9161. doi: 10.1007/s10570-019-02713-5 